\title{
On the origin of species: Factors shaping the establishment of infant's gut microbiota
}

Citation for published version (APA):

van Best, N., Hornef, M. W., Savelkoul, P. H. M., \& Penders, J. (2015). On the origin of species: Factors shaping the establishment of infant's gut microbiota. Birth Defects Research Part C-Embryo TodayReviews, 105(4), 240-251. https://doi.org/10.1002/bdrc.21113

Document status and date:

Published: 01/12/2015

DOI:

10.1002/bdrc.21113

Document Version:

Publisher's PDF, also known as Version of record

Document license:

Taverne

Please check the document version of this publication:

- A submitted manuscript is the version of the article upon submission and before peer-review. There can be important differences between the submitted version and the official published version of record.

People interested in the research are advised to contact the author for the final version of the publication, or visit the DOI to the publisher's website.

- The final author version and the galley proof are versions of the publication after peer review.

- The final published version features the final layout of the paper including the volume, issue and page numbers.

Link to publication

\footnotetext{
General rights rights.

- You may freely distribute the URL identifying the publication in the public portal. please follow below link for the End User Agreement:

www.umlib.nl/taverne-license

Take down policy

If you believe that this document breaches copyright please contact us at:

repository@maastrichtuniversity.nl

providing details and we will investigate your claim.
}

Copyright and moral rights for the publications made accessible in the public portal are retained by the authors and/or other copyright owners and it is a condition of accessing publications that users recognise and abide by the legal requirements associated with these

- Users may download and print one copy of any publication from the public portal for the purpose of private study or research.

- You may not further distribute the material or use it for any profit-making activity or commercial gain

If the publication is distributed under the terms of Article $25 \mathrm{fa}$ of the Dutch Copyright Act, indicated by the "Taverne" license above, 


\title{
Review
}

\section{On the Origin of Species: Factors Shaping the Establishment of Infant's Gut Microbiota}

\author{
Niels van Best ${ }^{1,2}$, Mathias W. Hornef ${ }^{1}$, Paul H. M. Savelkoul ${ }^{2,3,4}$, and John Penders ${ }^{\star 2,4}$
}

The human gut microbiota is a complex and dynamic ecosystem, which naturally lives in a symbiotic relationship with the host. Perturbations of the microbial composition (dysbiosis) and reduced diversity may promote disease susceptibility and recurrence. In contrast to the mature intestinal microbiota of healthy adults, which appears relatively stable over time, the infant's microbiome only establishes and matures during the first years of life. In this respect, early childhood seems to represent a crucial age-window in disease prevention, since microbial diversification and maturation of the microbiome primarily occurs during this period of life. A better understanding of ecological processes and pioneer consortia in microbial development is crucial, in order to support the development of a beneficial microbiota. Various deterministic and stochastic aspects seem to shape the microbiome in early life, including maternal, environmental, and host factors. Here, we review the current understanding of the origin of pioneer bacteria and the evolutionary factors that influence the development of the gut microbiota in infants. In addition, future perspectives, including manipulating and promoting the succession of initial bacteria during infancy, will be highlighted.

Birth Defects Research (Part C) 105:240-251, 2015. (c) 2015 Wiley Periodicals, Inc.

Key words: microbiome; ecology; antibiotics; cesarean section; diet

\section{Introduction}

The human gut microbiota is a complex and dynamic ecosystem consisting of hundreds to thousands of distinct bacterial species, as well as microbial eukaryotes, viruses, phages, and a limited number of Archaea, which naturally live in a symbiotic relationship with the host (Hooper and Gordon, 2001). In the past decades, the knowledge on the role of the gut microbiota and its development, as well as host-microbial interactions in human health and disease, has rapidly increased due to the advancement of modern molecular technologies. On one hand, the microbiota has a profound impact on its host by providing a competitive barrier against invading pathogens, utilizing undigested food components and producing essential metabolites, modulating immune responses, and stimulating intestinal maturation (Macpherson and Harris, 2004; Turnbaugh

The work of M.W.H. was supported by the priority program SPP1565 supported by the German Research Foundation (DFG).

${ }^{1}$ Institute of Medical Microbiology, RWTH University Hospital Aachen,

Aachen, Germany

${ }^{2}$ Department of Medical Microbiology, NUTRIM School of Nutrition and

Translational Research in Metabolism, Maastricht University Medical Centre+, Maastricht, The Netherlands

${ }^{3}$ Department of Medical Microbiology and Infection Control, VU University Medical Center Amsterdam, The Netherlands

${ }^{4}$ Department of Medical Microbiology, Caphri School for Public Health and Primary Care, Maastricht University Medical Centre+, Maastricht, The Netherlands

${ }^{*}$ Correspondence to: John Penders, Institute of Medical Microbiology, RWTH University Hospital Aachen, Aachen, Germany.

E-mail: j.penders@maastrichtuniversity.nl

Published online 26 November 2015 in Wiley Online Library (wileyonlinelibrary. com). Doi: 10.1002/bdrc.21113 et al., 2007). On the other hand, the host's immune system needs to tolerate the lumenal microbiota, but avoid their overgrowth and translocation of bacteria into the subepithelial tissue. As such, a perturbation of the microbial communities (dysbiosis) has the ability to promote disease susceptibility and progression. Indeed, alterations in microbiota composition and function have been associated with many diseases, including metabolic diseases (i.e. obesity, non-alcoholic fatty liver disease) (Turnbaugh et al., 2006; van Best et al., 2015) and immune-related diseases (i.e., inflammatory bowel disease, allergies) (Penders et al., 2007; Erickson et al., 2012; Rajilic-Stojanovic et al., 2015). Moreover, dysbiosis has also been linked to neoplastic diseases (Petra et al., 2014), mental disorders, and autismspectrum disorder (Mayer et al., 2015), although it has to be noted that for many of these diseases causality has still to be proven. The prevalence of many of these inflammatory noncommunicable diseases (NCDs) has increased rapidly over the past few decades, especially in western countries. As such, microbiota perturbations as a consequence of modern lifestyle are often held (in part) accountable for the rise in prevalence of NCD's (West et al., 2015).

Our knowledge on the intestinal microbial ecophysiology and the processes contributing to microbial dysbiosis has a direct impact on our ability to manage and maintain human health. In this respect, early childhood appears to be a crucial age-window, since diversification and maturation of the microbiota primarily occurs during this period of life. In contrast to the mature intestinal microbiota of healthy adults, which seems to be relatively stable over time, the infant microbiota waxes and wanes as diet changes and the host develops. Longitudinal studies monitoring the dynamics 
of the newborn microbiota (Favier et al., 2002; Palmer et al., 2007; Koenig et al., 2011) showed that periods of relative stability were interrupted by abrupt changes in microbial community structure and diversity. In some, but not all cases, these changes can be linked to preceding life events (e.g., weaning, fever, medication), indicating that both deterministic and stochastic aspects shape the microbiota in early life.

Here, we review the origin of pioneer bacteria and evolutionary factors that influence the development of the gut microbiota in infants. In addition, the potential of manipulating the microbiota for prevention and treatment of disorders in early life will be discussed.

\section{Factors Influencing the Intestinal Microbiota Development}

MATERNAL EXPOSURE

In utero transmission. Until recently, the initial microbial colonizers of the neonatal intestine were supposed to originate from the maternal perineal, vaginal, and fecal microbiota. However, the development of the microbiota composition might already start before birth by microbial transfer through the placental barrier. Recent sequencing studies revealed the presence of DNA derived from a wide variety of microbial taxa in the human placenta, umbilical cord blood, amniotic fluid, and meconium (Jimenez et al., 2008; DiGiulio, 2012; Funkhouser and Bordenstein, 2013; Aagaard et al., 2014). Although the presence of bacteria, such as Mycoplasma and Ureaplasma, have long been implicated in invasion of the amniotic cavity and detrimental health effects, such as preterm birth and necrotizing enterocolitis (Goldenberg and Culhane, 2003; OkogbuleWonodi et al., 2011; DiGiulio, 2012), these recent findings suggest that normal colonization may already start before rupture of membranes and birth. The placental microbiota has been suggested to resemble the oral microbiota from the Firmicutes, Tenericutes, Proteobacteria, Bacteroidetes, and Fusobacteria phyla, indicating a possible mode of bacterial transmission to the fetus (Aagaard et al., 2014). This evidence is, however, indirect as the investigators did not compare the placental and oral microbiota of the same subjects, but rather examined the oral microbiota in nonpregnant individuals. An experimental animal study using labeled Enterococcus faecium further evidenced that maternal bacteria can be transferred to fetuses in utero via the gastrointestinal tract (Jimenez et al., 2008). The underlying mechanism for this bacterial translocation remains unclear, but several possibilities have been proposed, i.e., via penetration of dendritic cells through the gut epithelium (Funkhouser and Bordenstein, 2013). Interestingly, bacterial translocation of the intestinal mucosa seems to be highly increased in pregnant mice, in comparison to nonpregnant animals (Perez et al., 2007).

Moreover, a number of studies detected a low abundant microbiota in the meconium of preterm and term infants using culture as well as molecular methods (Jimenez et al., 2008; Ardissone et al., 2014), implying that the neonatal gut may not be sterile at birth. Culture techniques revealed that Staphylococcus and Enterococcus predominated in the meconium (Jimenez et al., 2008). DNAbased techniques confirmed the predominance of these genera together with Enterobacteriaceae (Ardissone et al., 2014). The source of meconial bacteria remains unidentified, but they might have an intrauterine origin and be derived from swallowed amniotic fluid. The observation that the major genera observed in meconium are similar to those detected in amniotic fluid (DiGiulio, 2012) and less related to the bacteria prevailing in the maternal oral or vaginal microbiome, is supportive for an intrauterine origin (Ardissone et al., 2014).

Although these studies indicate that the neonatal gut might already become colonized with bacteria before birth, the majority of studies relied on culture-independent techniques, and therefore culture-based methods are ultimately required to establish a prepartum microbiota. The detected bacterial DNA sequences may originate from dead bacteria or free bacterial DNA in amniotic fluid, placenta, and meconium specimens. Moreover, not birth, i.e., transit through the birth canal, but rather rupture of the membranes, exposes the fetus to the environment and thus needs to be considered as a critical endpoint. Finally, the actual origin of these bacteria or bacterial remnants needs to be considered. Sampling of material from the same pregnant women at multiple sites may help to exclude local infection or sample contamination. Clearly, further research is necessary to clarify whether the potential in utero bacterial transmission of a low abundant inoculum truly contributes to the development of a healthy neonatal microbiome, immune maturation, and metabolism, or merely reflects the vulnerability that pregnancy inflicts to the maternal host.

Mode of delivery. The microbial colonization of the human intestine has traditionally been assumed to begin at birth and further develop during early life to a diverse and mature adult microbiota. Within a couple of days after birth, the neonatal intestine becomes rapidly colonized by maternal and environmental bacteria, strongly affected by the delivery mode (Penders et al., 2006; Dominguez-Bello et al., 2010). Infants born vaginally (i.e., passing the birth canal in intimate contact with the vaginal mucosa) display a microbiota similar to that of the maternal vagina, while infants delivered by cesarean (C-) section demonstrate a microbiota mainly consisting of commensal skin bacteria (Dominguez-Bello et al., 2010). Accordingly, the initial microbiota in vaginally delivered newborns is determined by the vaginal milieu of the mother, which is predominated by Lactobacillus, as well as by a high abundance of Prevotella and Sneathia spp. (Dominguez-Bello et al., 2010; Romero et al., 2014; MacIntyre et al., 2015). Infants born 
by $\mathrm{C}$-section seem to have, in general, delayed and decreased colonization of Bifidobacterium spp. and Bacteroides spp. and increased rates of Clostridium difficile and typical skin bacteria such as Staphyloccocus, Corynebacterium, and Propionibacterium spp,. compared with vaginally delivered infants (Penders et al., 2006; Dominguez-Bello et al., 2010; Fallani et al., 2010; Azad et al., 2013a; Jakobsson et al., 2014; Backhed et al., 2015). Increased rates of $C$. difficile may result from environmental exposure, since $C$. difficile spores can be found widespread in the hospital setting. Consistently, $C$. difficile has been shown to be more prevalent in neonates delivered in the hospital, as compared with those born at home. Moreover, the risk of colonization by this bacterium increased with each additional day of hospitalization (Penders et al., 2006). Lacking the inoculum of the maternal microbiota, the hospital environment and staff may represent an important source of bacteria colonizing the intestinal tract of $\mathrm{C}$-section delivered infants. Longitudinal studies showed that the delayed and altered colonization pattern in C-section delivered infants persisted at least to the age of one year (Jakobsson et al., 2014; Backhed et al., 2015), and even after 7 years of age minor differences could be detected (Salminen et al., 2004). However, a potential functional impact on infant's development and wellbeing has not been examined. In addition, Salminen et al. (2004) visualized specific bacteria by fluorescent in situ hybridization (FISH), and therefore comprehensive data on a fully established microbiota in later childhood in relation to the mode of delivery are still lacking.

Given the profound impact of the mode of delivery on the establishment of the neonatal microbiome and its potential consequences, the continuously increasing Csection rates in many parts of the world are worrisome. Recent findings, based on the ecological association of cesarean delivery rates and perinatal outcomes from 19 developed countries (Ye et al., 2014), corroborated previous suggestions of the World Health Organization (WHO) expert panel that population-level C-section delivery rates above 10 to $15 \%$ are hardly justified by medical reasoning. Yet, during the past decades, $\mathrm{C}$-section rates have rapidly increased far above 10 to $15 \%$ in many countries, mainly attributable to nonevidence-based indications, professional convenience, maternal request, and overmedication of child-birth (Robson et al., 2013). In many European countries, the United States, and the larger cities in China and Australia, rates have risen up to above $30 \%$, while rates as high as $45 \%$ have been reported for Brazil (Gibbons et al., 2010).

The increased risk of obesity (Kuhle et al., 2015) and childhood asthma (Huang et al., 2014) in children born by C-section has been attributed to an altered intestinal colonization pattern in these children. Indeed, within the context of two birth cohort studies, mode of delivery was shown to strongly impact the infant microbiota and affect the risk of allergies and asthma (Penders et al., 2014). Moreover, the decreased abundance of Bifidobacterium and higher numbers of $S$. aureus in the intestine during early life has been associated with subsequent overweight and obesity in childhood (Kalliomäki et al., 2008), emphasizing the critical role of the first exposure.

\section{INFANT FEEDING}

Breastfeeding plays both directly, by containing living bacteria, and indirectly, by providing prebiotic nutrients and bioactive components, an important role in the establishment of the neonatal microbiota. The first abundant bacteria of the newborn's gut are generally facultative anaerobes, such as Escherichia, Streptococcus, and Enterococcus, probably due to the oxygen-rich environment in the neonatal gut lumen (Avershina et al., 2014; Backhed et al., 2015). Although some studies observed a gradual shift in microbial composition and increase in diversity (Koenig et al., 2011), other studies demonstrated chaotic shifts over time, including a transient drop in diversity within the first week of life (Yatsunenko et al., 2012; Avershina et al., 2014). The lack of extensive bacterial competition results in high population levels in neonates, exceeding the numbers found in adults (Stark and Lee, 1982). Facultative anaerobes utilize oxygen and thereby create a reduced environment, making it more favorable for subsequent colonization of strict anaerobe bacteria. Therefore, the microbiota development continues in the first weeks, with proliferation of strictly anaerobic bacteria that are able to convert lactic acid derived from to the milk-rich diet. Infants that are exclusively breast-fed in general harbor a microbiota dominated by bifidobacteria, while exclusively formula-fed infants harbor a more diverse microbiota with increased abundance of Escherichia coli, Clostridia, and Bacteroides (Penders et al., 2006; Fallani et al., 2010; Bezirtzoglou et al., 2011; Azad et al., 2013a; Backhed et al., 2015). A recent study revealed by deep sequencing that formula-fed infants displayed enhanced populations of Ganulicatella, Citrobacter, Enterobacter, and Bilophila, whereas breast-fed infants showed increased Lactobacillus species apart from bifidobacteria (Backhed et al., 2015). The beneficial effects of human milk oligosaccharides (HMOs), a structurally diverse family of unconjugated glycans that are highly abundant in human milk and absent in formula nutrition, might cause the differences in the microbiota composition between formula-fed and breast-fed infants. The maternal HMO's have a prebiotic role and promote the growth of Bifidobacterium, increase microbial diversity, and are able to protect the infant against a number of enteropathogenic microorganisms (Zivkovic et al., 2011). The quantity and composition of these oligosaccharides varies over the course of the lactation period, but also between individual women, mainly driven by their genotype (Bode, 2012; CabreraRubio et al., 2012). 
Additionally, breastmilk contains other immunologic compounds, i.e., IgA, lactoferrin, and lysozyme (Pacheco et al., 2015). All these factors are able to interact with pathogens, reduce their viability, and prevent their attachment to the intestinal mucosal surface, assisting the expansion of commensal bacteria. Therefore, factors such as the IgA repertoire might shape a distinct neonatal microbiota (Rogier et al., 2014). Vitamin D, which is part of infant formulas and a recommended supplement in breastfed infants, is another compound that has been suggested to influence the developing microbiome (Ly et al., 2011), given its role in the development of regulatory Tcells and dendritic cells (Griffin et al., 2003). Direct evidence, however, is limited to a single study correlating a lower vitamin D intake to a different microbiota composition in adult African Americans, as compared with Caucasian Americans (Volker et al., 2009). The results need to be confirmed in larger longitudinal studies.

Interestingly, recent studies also revealed the presence of a microbiota in maternal breastmilk. With concentrations of $10^{2}$ to $10^{4}$ viable bacterial per mL breast milk, an exclusively breastfed infant will consume the significant amount of about $10^{5}$ to $10^{7}$ commensal bacteria per day (Heikkila and Saris, 2003). A study on variations in the breastmilk microbiome over the course of lactation indicated that staphylococci, streptococci, and lactobacilli derived from the milk ducts and skin predominated in colostrum, whereas after six months a higher abundance of oral taxa, including Veillonella and Prevotella, were found in breastmilk (Cabrera-Rubio et al., 2012). Other studies hypothesize an entero-mammary pathway, postulating that maternal gut bacteria could translocate through the intestinal epithelial barrier, migrate to the mammary gland, and subsequently become secreted with the milk and colonize the breastfed neonate (Fernandez et al., 2013). Using a combination of culture-dependent and independent methods, Jost et al. (2014) examined whether viable strains of gut-associated obligate anaerobes were shared between the maternal and neonatal gut microbiota and transferred via breastfeeding. Next to facultative anaerobes, obligate anaerobic genera, including Bifidobacterium, Bacteroides, and Clostridia were detected in maternal faeces, breast milk, and neonatal feces. Moreover, pulsefield gel electrophoreses (PFGE) of Bifidobacterium breve isolates from maternal feces, breast milk, and neonatal feces of a single mother-child pair, revealed identical restriction profiles, suggesting transfer of these strains from the maternal to the neonatal gut via breast milk (Jost et al., 2014). Alternatively, the maternal gut might represent a common source for both the mammary skin/gland duct bacteria as well as the neonatal intestine.

The third stage in the development of the microbiota is defined by alterations of the microbial composition and function, due to the introduction of solid food 4 to 6 months postpartum (weaning). Upon weaning, the microbiota functionally maturates by a decrease in the relative abundance of genes involved in the degradation of sugars from breast milk and towards enrichment of genes involved in the degradation of complex sugars and starch. The major pectin-degrading enzyme, pectinesterase, has been shown to be enriched in infants by the age of 12 months, likely in response to the increased intake of foods rich in pectin by this age (Backhed et al., 2015). The particular effects of solid foods, however, strongly depend on the geography linked to major differences in dietary habits around the world. In western countries, the introduction of a more complex diet rapidly increases the levels of Bacteroides and Clostridium, alters the lactobacilli communities, and decreases the Bifidobacterium levels (Yatsunenko et al., 2012; Avershina et al., 2014; Bergstrom et al., 2014; Backhed et al., 2015). The intestinal microbiota closely resembles the diverse adult-like composition at the age of three years (Yatsunenko et al., 2012; Bergstrom et al., 2014), even though older data indicated that microbial stability is already achieved at the age of 1 year (Palmer et al., 2007; Koenig et al., 2011). Interestingly, a longitudinal study of 98 Swedish mothers and their infants also compared the bacterial composition between the introduction of solid food with and without continuation of breastfeeding (Backhed et al., 2015). This comparison suggested that the cessation of breastfeeding, rather than the introduction of solid food, represents the major factor driving the microbiota composition towards a more adult-like ecology. Altogether, these data emphasize the role of breastmilk and diet in the succession and shaping of the intestinal microbiota in early life.

\section{ENVIRONMENTAL EXPOSURE}

Besides the influence of the mother and the associated exposures during delivery, the child's living environment, including the indoor home environment, pets, and older siblings as sources for microbial exposure, has been shown to affect the intestinal microbial development. A recent longitudinal study by the Home Microbiome Consortium, showed substantial interaction among the human, home, and pet microbiota (Lax et al., 2014). Moreover, strikingly similar dynamics in the development of the enteric microbiota of a fraternal twin pair, including the coincidental transient appearance of specific organisms in both twins, suggest that stochastic environmental influences can also play an important role during the assembly of microbial communities (Palmer et al., 2007).

Siblings. Singletons seem to have a distinct colonization pattern in comparison with infants that grow up together with older siblings, although data are scarce and not always consistent. The Canadian Healthy Infant Longtudinal Development (CHILD) cohort showed a lower abundance of $C$. difficile and its family Peptostreptococcaceae (Azad et al., 2013a), whereas a German study reported decreased colonization rates of Clostridium cluster I in 
newborns with older siblings, as compared with singletons (Penders et al., 2013). The higher levels of Clostridium species in singletons are in accordance with another study comprising three European birth cohorts (Adlerberth et al., 2007). A decrease in bifidobacteria in infants without older siblings has also been reported (Penders et al., 2006). Moreover, a higher microbial richness and diversity has recently been observed in infants with older siblings (Laursen et al., 2015). Altogether, these data suggest that firstborn infants harbor a less mature microbiota than infants with older siblings at a certain age, which may impact the risk on allergies (Penders et al., 2014). Whether this occurs due to direct or indirect bacterial transfer between siblings or due to other factors, such as altered vaginal microbiota and breastmilk composition in multiparous, as compared with primiparous women, remains to be elucidated.

Pets and house dust. There is limited data available on the role of in-house microbial exposure in humans, but an association between the bacteria found in house dust and in children has recently been reported (Konya et al., 2014). Moreover, Azad et al. (2013b) observed that the presence of pets might affect infant colonization, in contrast to studies that did not find a relation between the presence of household pets and the human gut microbiota (Penders et al., 2006; Konya et al., 2014). Pets might also cause the occurrence of certain bacteria in house dust and transfer of specific communities to humans. Infants living with pets showed mainly increased Peptostrepococcaceae (C. difficile) and decreased abundance of bifidobacteria at 4 months of age. The discrepancy between studies might indicate the minor role of pets, and more research is necessary to confirm the association and identify transfer pathways.

Geography. The microbial ecology also differs between infants from various geographical habitats. Children born in developing countries are characterized by enhanced levels of Prevotella and decreased abundance of Bacteroides in early life, as compared with infants living in western countries (De Filippo et al., 2010; Grzeskowiak et al., 2012; Yatsunenko et al., 2012; Clemente et al., 2015). The geographical variation in colonization patterns might mainly result from differences in diet, as segregation of the microbiota composition into distinct geographic clusters becomes apparent only after weaning, i.e., the start of consumption of solid food (De Filippo et al., 2010). The food in developing countries is low in fat and protein, and rich in fibers, compared with the typical western diet. In addition, the advanced sanitary conditions in western countries, compared with a less advanced infrastructure and crowded settings in developmental countries, could also influence differences in indigenous microbial community structures. Furthermore, intra-continental differences between western countries have also been reported with lower proportions of Bifidobacterium and higher proportions of Akkermansia, Clostridium, and Bacteroides in German infants than Finish infants (Grześkowiak et al., 2012). The latter is in line with another European study that showed overrepresentation of Bifidobacterium in Northern European infants, whereas increased diversity was detected in Southern European infants (Fallani et al., 2010). However, whether these differences are driven by environmental exposure, genetic make-up, or dietary factors is currently unclear.

\section{HOST GENETICS}

Host genotype is another factor with a major impact on the microbial composition, as shown by studies comparing the microbiota of individuals with varying degrees of relatedness, in particular monozygotic as compared with dizygotic twins. Several studies using traditional microbial fingerprinting techniques in both children and adults reported host genetic effects on the microbiota composition (Van de Merwe et al., 1983; Zoetendal et al., 2001; Stewart et al., 2005). A recent study analyzing the microbiota development by next-generation sequencing in three dichorionic triplet sets showed that at the age of one month the monozygotic twin pairs shared a more similar microbiota, as compared with their fraternal siblings. By month 12 however, the profile became more uniform between the three infants, suggesting that host genetics initially play a significant role, while environmental determinants dominate by the age of 12 months (Murphy et al., 2015).

By far the most comprehensive study to date examined the influence of host genetics on the microbiota using fecal samples of 416 pairs of adult twins from the TwinsUK cohort (Goodrich et al., 2014). The microbiota were more similar between twin pairs as compared with unrelated individuals, and were also more similar between monozygotic twins than dizygotic twins. By estimating the heritability of individual microbial taxa, it was shown that the community structure within the phylum Bacteroidetes was mainly shaped by environmental factors. Heritability appeared strongest for families within the phylum Firmicutes, including Ruminococcaceae and Lachnospiraceae, with Christensenellaceae being the most heritable family. Christensenellaceae formed a co-occurrence network with other microbes, such as methanogenic Archaea, and was associated with lean BMI. The functional relevance was illustrated by subsequent transfer of Christensenellaceae species to germ-free mice resulting in reduced body weight. Interestingly, the authors were able to validate their findings of heritability of the above-mentioned bacterial families by applying their statistical models to the data of two studies that had previously failed to reveal significant genotype effects on the microbiota (Turnbaugh et al., 2009; Yatsunenko et al., 2012). 
Furthermore, studies using a candidate gene approach examined the involvement of individual genes. For instance, individuals that have a functional fucosyltransferase 2 (FUT2) gene, referred as secretors, displayed different microbial communities compared with non-secretors (Rausch et al., 2011). The secretor-status regulates the existence of mucosal $\mathrm{ABH}$ and Lewis blood group antigens, but also the glycosylation of the intestinal mucus and breastmilk oligosaccharides. In addition, fucosyltransferase 3 (FUT3) produces the Lewis A antigens in nonsecretors and B antigens in secretors. The profiles of the human milk oligosaccharides have been shown to differ in accordance to maternal secretor status, as well as Lewis blood group antigen, due to genetic variations of FUT2 and FUT3, respectively (Totten et al., 2012). In accordance, a recent study observed earlier colonization and higher levels of bifidobacteria in breastfed infants of maternal secretors (Lewis et al., 2015). Many other candidate genes have been suggested to influence microbiota composition. Among them in particular are genes encoding immune mediators, such as NOD2 and $M E F V$, reviewed in detail elsewhere (Marietta et al., 2015). The nucleotide-binding oligomerization domain 2 (NOD2) receptor recognizes bacterial cell wall-derived muramyl dipeptide fragments and regulates the release of $\alpha$-defensins by Paneth cells. Consistent with an influence of enteric antimicrobial peptides on microbiota composition (Salzman et al., 2010), expression of NOD2 has been shown to affect the microbial ecology in mice (Petnicki-Ocwieja et al., 2009). In humans, NOD2 mutations are a risk factor for the development of inflammatory bowel disease and are associated with microbial dysbiosis (Frank et al., 2011). Mutations in the human gene $M E F V$ that encodes pyrin result in familial Mediterranean fever, an autoimmune disease with uncontrolled interleukin (IL)-1 release. Mutations in MEFV are associated with changes in the gut microbiota, including lower bacterial diversity (Khachatryan et al., 2008). These findings highlight that the genotype of the host influences the intestinal microbiota composition, which in turn, may influence host metabolism.

\section{ANTIBIOTICS}

Although the majority of common pediatric infections, e.g., upper respiratory tract infections, are self-limiting and caused by viruses, large volumes of broad-spectrum antibiotics are still being prescribed for these conditions in primary care settings (Hersh et al., 2011), with little evidence for clinical benefit (Smith et al., 2014). Next to being the main factor driving the development and dissemination of antimicrobial resistance, (over)use of antibiotics has profound and direct effects on the indigenous microbiota. The exact effects on the microbiota composition are dependent on the specific type of antibiotic, but also differ between individuals, as was demonstrated in a study on the effects of ciprofloxacin treatment in healthy volunteers
(Dethlefsen et al., 2008). This latter study showed that the microbiota is relatively resilient to perturbations induced by a single course of antibiotics. A subsequent study, however, indicated that the microbiota composition may permanently shift to an alternative state, upon multiple courses of ciprofloxacin (Dethlefsen and Relman, 2011). In newborns exposed to antibiotics, obligate anaerobic genera, such as Bacteroides spp. and Bifidobacterium spp. and Lactobacillus spp., are depleted, whereas levels of Proteobacteria increase (Penders et al., 2006; Savino et al., 2011; Fouhy et al., 2012). Not surprisingly, maternal antibiotic use during pregnancy or breastfeeding has also been shown to affect the infant's microbiota composition with decreased levels of Bacteroides spp. and Atopbium spp. (Fallani et al., 2010). Interestingly, the maternal vaginal microbiota is affected by antibiotic use during pregnancy, which might contribute to a distinct neonatal microbiome (Stokholm et al., 2014).

To date, few studies have examined the effect of intrapartum antibiotic prophylaxis (IAP), a practice routinely used in pregnant women positive for group B streptococci (GBS) on the vaginal mucosa, on the establishment of the neonatal microbiome. GBS represents the most important causative agent of neonatal sepsis with often fatal outcome. In the absence of an available vaccine, prophylactic antibiotic treatment represents the recommended measure to prevent infection. Two recent studies, quantifying a selected group of bacterial genera, revealed a decrease in bifidobacterial abundance, as well as a shift in bifidobacterial species composition, in newborns whose mothers did receive IAP, as compared with newborns without maternal IAP exposure (Aloisio et al., 2014; Corvaglia et al., in press). In addition, newborns are (indirectly) exposed to antibiotics during labor, as prophylaxes prior to C-section. The effects on the neonatal microbiome have, however, not been characterized.

Although there is little direct evidence from human studies, it is highly likely that the developing infant microbiota is far less resilient to antibiotic-induced perturbations than the microbiota of adults. This is supported by a recent study mimicking pediatric antibiotic use in a mouse-model by providing early-life administration of antibiotics at therapeutic doses ( $\beta$-lactam and macrolides). Progressive and long-term alterations in the gut microbiota composition and metagenomic content were observed, varying with the number of courses and class of antibiotic treatment (Nobel et al., 2015). Moreover, murine antibiotic exposure in early life showed an altered host metabolism regarding energy harvesting and fat storage (Cox et al., 2014; Nobel et al., 2015). Therefore, antibiotic use during infancy has been suggested to increase subsequent weight gain and enhance the risk of childhood overweight (Ajslev et al., 2011; Trasande et al., 2013; Cox and Blaser, 2015). The latter studies indicate that perturbation of the microbiota in early life results in different metabolic 
effects even in the presence of a similar diet. The lack of acquisition or the extinction of specific species during the neonatal period might therefore represent a critical factor, emphasizing the key role of pioneer bacteria.

WINDOW OF OPPORTUNITY FOR MICROBIOTA MANIPULATION

The therapeutic manipulation of the gut microbiota is promising, since the microbiota plays a crucial role in human health. For instance, fecal transplantation is a procedure that has recently raised a lot of scientific awareness and many intervention studies are currently ongoing to treat a variety of diseases. However, this method has so far only been clinically accepted as an effective method to eradicate $C$. difficile infection (Drekonja et al., 2015). Moreover, numerous randomized placebo-controlled trials have been conducted on the efficacy of pre- and probiotic supplementation in pregnant women and newborns in the primary prevention of diseases, such as necrotizing enterocolitis (NEC), atopic diseases, and (extra)intestinal infections. For most clinical outcomes the efficacy of preand probiotic supplementation is inconclusive, although there is evidence that probiotics might reduce the risk of atopic eczema in infants (Cuello-Garcia et al., in press) and NEC in preterm neonates (AlFaleh and Anabrees, 2014). Yet, there is great heterogeneity between studies in terms of the probiotic strains used, dosing, duration, and timing of probiotic therapy. The understanding and selection of the appropriate probiotics is crucial and should be determined by fundamental host-microbiota interactions. Importantly, a better knowledge on the bacteria that are essential for specific health effects might also stimulate the use of selected diets or drugs as a way to modify the microbiota. Further research is necessary to identify not only beneficial bacteria, but also other microbes, such as Archaea and viruses, and their interactions. This may include approaches that use phage therapy as a tool against specific bacteria or pathogens (Viertel et al., 2014).

In addition, a better understanding of ecological processes and pioneer consortia in microbial development will be crucial to design efficacious therapies and standardized manipulations. The microbial maturation relies on the succession of initial bacteria during infancy, making early life a particular suitable period to modify the microbiota in favor of health-promoting bacteria. The identification of specific neonatal populations that will benefit most from microbiota manipulation could also be relevant in terms of personalized strategies, e.g., following C-section delivery, intra-partum antibiotic prophylaxis or neonatal infections. In this respect, trials are under way in which newborns delivered via $\mathrm{C}$-section are being exposed to the maternal vaginal microbiota directly after birth. However, further studies are needed to unravel the critical shaping factors of intestinal bacteria in early life, in order to support future targeted microbial therapies to reduce the risk of disease.
CONCLUSION AND FUTURE PERSPECTIVES

Our knowledge on the maturation dynamics of the gut microbiota during infancy has advanced considerably during the past decades. The microbiota develops from the initial inoculum to a complex and diverse ecosystem during the first years of life and is influenced by a large number of perinatal environmental and maternal factors as summarized in Fig. 1. The colonization of essential bacteria in the neonatal gut is important, since their lack might result in increased risk of metabolic or immuneassociated disorders, such as obesity or allergy, respectively. The establishment of the microbiota and its function during early life is strongly affected by the mode of delivery, breastmilk versus formula, and diet. Studies also emphasize the destructive role of antibiotic exposure in the succession of initial bacteria. In addition, the child's living location, including the indoor home environment, the presence of pets and older siblings as sources for microbial exposure, has been suggested to impact the infant microbiota. Further studies highlighted the critical role of the host's genotype in the intestinal microbiota composition, although these results were mostly derived from animal studies. Importantly, besides the determinants that select or shape the microbiota, the order of acquiring specific bacteria-the historical contingencyshould also be taken into account. The historical contingency might be crucial because it defines how bacteria interact with each other and with the host, and may in turn influence the entire community (Dethlefsen et al., 2006). However, the order of microbial arrivals is mainly stochastic and unknown, resulting in highly unpredictable microbial interactions. Therefore, experimental studies providing insight in the extent to which historical contingency contributes to microbial assembly are pivotal. Large-scale birth cohort studies generally assess the growth or stability of the microbiome by sampling at intervals ranging from months or years, but whether these dynamics sufficiently reflect the variability over a shorter period is unclear. As such, a regimen of more regular sampling moments (weekly or even daily) within the context of longitudinal birth cohort studies is warranted. Moreover, further research is needed to unravel the functional and metabolic development of the neonatal microbiome. The integration of metatranscriptomics and metabolomics in future longitudinal human studies will greatly improve our understanding of the role of the infant's microbiome. It will also be crucial to determine the impact of early microbial changes on the incidence and severity of certain diseases in infancy as well as in later life. Consequently, new longitudinal studies should define in more detail the host-microbe interactions in regard to infant's health and provide sufficient follow-up to monitor the onset of non-communicable diseases. For instance, C-section may have a long-term impact on child's health, regarding immune-mediated disorders 


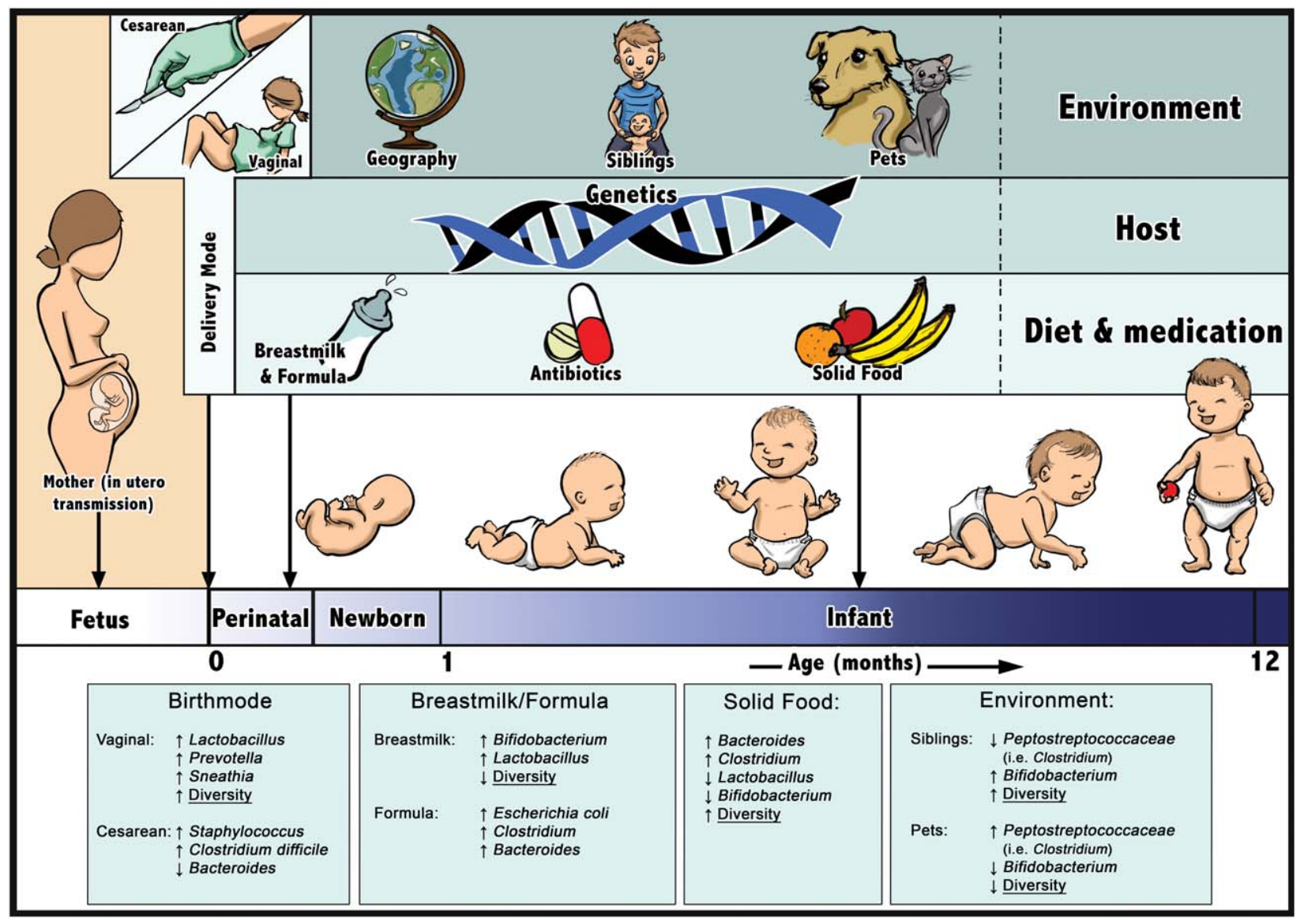

FIGURE 1. Main maternal, environmental, and host determinants that impact the establishment of the intestinal microbiota during the first year of life. A nonexclusive listing of bacterial taxa affected by these determinants is depicted at the bottom of the figure. Note: the timeline is colored ranging from white to blue corresponding to a change in microbial diversity from low to high, respectively. Perinatal starts at rupture of the membranes.

(Decker et al., 2010; Penders et al., 2014), as well as increased BMI in offspring and obesity in adulthood (Darmasseelane et al., 2014). The latter emphasizes the crucial role of the first inoculum, and suggests that the vaginal microbiota has the ability to induce metabolic and immune maturation in the newborn over a prolonged time period. Insight into the multifaceted interactions between the microbiota and ecological as well as host factors are still in its infancy, and need to be expanded to facilitate therapeutic or prophylactic microbiota manipulations. Altogether, the overall challenge will be to promote certain bacterial populations in early life, prevent their eradication by antibiotics, and in this way prevent the development of non-communicable diseases.

\section{Acknowledgment}

The authors are thankful to Mayk Lucchesi for figure designing and formatting.

\section{Emphasize Box}

Data from human infants are commonly based on stool samples which deviate considerably from colonic mucosal microbiota (Zoetendal et al., 2002). Recent high-throughput microbial profiling also showed that the mucosal microbiota even varies along the complete length of the gut (Zhang et al., 2014). Therefore, data from animals of small intestinal and colonic microbiota provides additional insight on the development, as well as specific host-microbe interactions. Although mice and rats have an increased exposure to environmental and fecal microbiota, the essence of the initial succession of gut bacteria in rodents reflects that in humans. In mice, the neonatal gut is mainly dominated by Lactobacillaceae, Streptococcus and Enterobacteriaceae (Pantoja-Feliciano et al., 2013; Del Chierico et al., 2014). The colon in mice is first established by bacteria that resemble the vaginal microbiota, followed by a decrease in microbial diversity and shift in dominance from Streptococcus to Lactobacillus (PantojaFeliciano et al., 2013). The microbial abundance seems to be related to the anatomical complexity of the intestine, resulting in very low 
microbial diversity in a simple and small gut after birth (Del Chierico et al., 2014). After the introduction of solid food, the murine microbiota increases in strictly anaerobes such as Bacteroides, Lachnospiraceae, and Clostridium. Altogether, animal studies further indicate that the microbiota development is multiple-phasic and is pivotal in unraveling host-microbiota relationships.

\section{References}

Aagaard K, Ma J, Antony KM, et al. 2014. The placenta harbors a unique microbiome. Sci Transl Med 6:237ra265.

Adlerberth I, Strachan DP, Matricardi PM, et al. 2007. Gut microbiota and development of atopic eczema in 3 European birth cohorts. J Allergy Clin Immunol 120:343-350.

Ajslev TA, Andersen CS, Gamborg M, et al. 2011. Childhood overweight after establishment of the gut microbiota: the role of delivery mode, pre-pregnancy weight and early administration of antibiotics. Int J Obesity 35:522-529.

AlFaleh K, Anabrees J. 2014. Probiotics for prevention of necrotizing enterocolitis in preterm infants. Evidence Based Child Health Cochrane Rev J 9:584-671.

Aloisio I, Mazzola G, Corvaglia LT, et al. 2014. Influence of intrapartum antibiotic prophylaxis against group B Streptococcus on the early newborn gut composition and evaluation of the antiStreptococcus activity of Bifidobacterium strains. Appl Microbiol Biotechnol 98:6051-6060.

Ardissone AN, de la Cruz DM, Davis-Richardson AG, et al. 2014. Meconium microbiome analysis identifies bacteria correlated with premature birth. PLoS One 9:e90784.

Avershina E, Storro 0, Oien T, et al. 2014. Major faecal microbiota shifts in composition and diversity with age in a geographically restricted cohort of mothers and their children. FEMS Microbiol Ecol 87:280-290.

Azad MB, Konya T, Maughan H, et al. 2013a. Gut microbiota of healthy Canadian infants: profiles by mode of delivery and infant diet at 4 months. CMAJ 185:385-394.

Azad MB, Konya T, Maughan H, et al. 2013b. Infant gut microbiota and the hygiene hypothesis of allergic disease: impact of household pets and siblings on microbiota composition and diversity. Allergy Asthma Clin Immunol 9:15.

Backhed F, Roswall J, Peng Y, et al. 2015. Dynamics and stabilization of the human gut microbiome during the first year of life. Cell Host Microbe 17:690-703.

Bergstrom A, Skov TH, Bahl MI, et al. 2014. Establishment of intestinal microbiota during early life: a longitudinal, explorative study of a large cohort of Danish infants. Appl Environ Microbiol 80:2889-2900.

Bezirtzoglou E, Tsiotsias A, Welling GW. 2011. Microbiota profile in feces of breast- and formula-fed newborns by using fluorescence in situ hybridization (FISH). Anaerobe 17:478-482.
Bode L. 2012. Human milk oligosaccharides: every baby needs a sugar mama. Glycobiology 22:1147-1162.

Cabrera-Rubio R, Collado MC, Laitinen K, et al. 2012. The human milk microbiome changes over lactation and is shaped by maternal weight and mode of delivery. Am J Clin Nutr 96:544-551.

Clemente JC, Pehrsson EC, Blaser MJ, et al. 2015. The microbiome of uncontacted Amerindians. Sci Adv 1(3):e1500183.

Corvaglia L, Tonti G, Martini S, et al. Influence of intrapartum antibiotic prophylaxis for group B Streptococcus on gut microbiota in the first month of life. J Pediatr Gastroenterol Nutr. doi: 10.1097/MPG.0000000000000928.

Cox LM, Blaser MJ. 2015. Antibiotics in early life and obesity. Nat Rev Endocrinol 11:182-190.

Cox LM, Yamanishi S, Sohn J, et al. 2014. Altering the intestinal microbiota during a critical developmental window has lasting metabolic consequences. Cell 158:705-721.

Cuello-Garcia CA, Brozek JL, Fiocchi A, et al. 2015. Probiotics for the prevention of allergy: a systematic review and meta-analysis of randomized controlled trials. J Allergy Clin Immunol 136:952961.

Darmasseelane K, Hyde MJ, Santhakumaran S, et al. 2014. Mode of delivery and offspring body mass index, overweight and obesity in adult life: a systematic review and meta-analysis. PLoS One 9:e87896.

De Filippo C, Cavalieri D, Di Paola M, et al. 2010. Impact of diet in shaping gut microbiota revealed by a comparative study in children from Europe and rural Africa. Proc Natl Acad Sci USA 107:14691-14696.

Decker E, Engelmann G, Findeisen A, et al. 2010. Cesarean delivery is associated with celiac disease but not inflammatory bowel disease in children. Pediatrics 125:e1433-e1440.

Del Chierico F, Petrucca A, Mortera SL, et al. 2014. A metaproteomic pipeline to identify newborn mouse gut phylotypes. J Proteomics 97:17-26.

Dethlefsen L, Eckburg PB, Bik EM, Relman DA. 2006. Assembly of the human intestinal microbiota. Trends Ecol Evol 21:517523.

Dethlefsen L, Huse S, Sogin ML, Relman DA. 2008. The pervasive effects of an antibiotic on the human gut microbiota, as revealed by deep 16S rRNA sequencing. PLoS Biol 6:e280.

Dethlefsen L, Relman DA. 2011. Incomplete recovery and individualized responses of the human distal gut microbiota to repeated antibiotic perturbation. Proc Natl Acad Sci USA 108(Suppl 1): 4554-4561.

DiGiulio DB. 2012. Diversity of microbes in amniotic fluid. Semin Fetal Neonat Med 17:2-11.

Dominguez-Bello MG, Costello EK, Contreras M, et al. 2010. Delivery mode shapes the acquisition and structure of the initial 
microbiota across multiple body habitats in newborns. Proc Natl Acad Sci USA 107:11971-11975.

Drekonja D, Reich J, Gezahegn S, et al. 2015. Fecal microbiota transplantation for Clostridium difficile infection: a systematic review. Ann Inter Med 162:630-638.

Erickson AR, Cantarel BL, Lamendella R, et al. 2012. Integrated metagenomics/metaproteomics reveals human host-microbiota signatures of Crohn's disease. PLoS One 7:e49138.

Fallani M, Young D, Scott J, et al. 2010. Intestinal microbiota of 6-week-old infants across Europe: geographic influence beyond delivery mode, breast-feeding, and antibiotics. J Pediatr Gastroenterol Nutr 51:77-84.

Favier CF, Vaughan EE, De Vos WM, Akkermans ADL. 2002 Molecular monitoring of succession of bacterial communities in human neonates. Appl Environ Microbiol 68:219-226.

Fernandez L, Langa S, Martin V, et al. 2013. The human milk microbiota: origin and potential roles in health and disease. Pharmacol Res 69:1-10.

Fouhy F, Guinane CM, Hussey S, et al. 2012. High-throughput sequencing reveals the incomplete, short-term recovery of infant gut microbiota following parenteral antibiotic treatment with ampicillin and gentamicin. Antimicrob Agents Chemother 56:5811-5820.

Frank DN, Robertson CE, Hamm CM, et al. 2011. Disease phenotype and genotype are associated with shifts in intestinalassociated microbiota in inflammatory bowel diseases. Inflamm Bowel Dis 17:179-184.

Funkhouser LJ, Bordenstein SR. 2013. Mom knows best: the universality of maternal microbial transmission. PLoS Biol 11:e1001631.

Gibbons L, Belizán JM, Lauer JA, et al. 2010. The global numbers and costs of additionally needed and unnecessary caesarean sections performed per year: overuse as a barrier to universal coverage. World Health Report 30:1-31.

Goldenberg RL, Culhane JF. 2003. Infection as a cause of preterm birth. Clin Perinatol 30:677-700.

Goodrich JK, Waters JL, Poole AC, et al. 2014. Human genetics shape the gut microbiome. Cell 159:789-799.

Griffin MD, Xing N, Kumar R. 2003. Vitamin D and its analogs as regulators of immune activation and antigen presentation. Annu Rev Nutr 23:117-145.

Grzeskowiak L, Collado MC, Mangani C, et al. 2012. Distinct gut microbiota in southeastern African and northern European infants. J Pediatr Gastroenterol Nutr 54:812-816.

Grześkowiak L, Grönlund MM, Beckmann C, et al. 2012. The impact of perinatal probiotic intervention on gut microbiota: double-blind placebo-controlled trials in Finland and Germany. Anaerobe 18:7-13.

Heikkila MP, Saris PE. 2003. Inhibition of Staphylococcus aureus by the commensal bacteria of human milk. J Appl Microbiol 95: 471-478.
Hersh AL, Shapiro DJ, Pavia AT, Shah SS. 2011. Antibiotic prescribing in ambulatory pediatrics in the United States. Pediatrics 128:1053-1061.

Hooper LV, Gordon JI. 2001. Commensal host-bacterial relationships in the gut. Science 292:1115-1118.

Huang L, Chen Q, Zhao Y, Wang W. 2014. Is elective cesarean section associated with a higher risk of asthma? A meta-analysis. J Asthma 52:16-25.

Jakobsson HE, Abrahamsson TR, Jenmalm MC, et al. 2014. Decreased gut microbiota diversity, delayed Bacteroidetes colonisation and reduced Th1 responses in infants delivered by caesarean section. Gut 63:559-566.

Jimenez E, Marin ML, Martin R, et al. 2008. Is meconium from healthy newborns actually sterile? Res Microbiol 159:187-193.

Jost T, Lacroix C, Braegger CP, et al. 2014. Vertical motherneonate transfer of maternal gut bacteria via breastfeeding. Environ Microbiol 16:2891-2904.

Kalliomäki M, Collado MC, Salminen S, Isolauri E. 2008. Early differences in fecal microbiota composition in children may predict overweight. Am J Clin Nutr 87:534-538.

Khachatryan ZA, Ktsoyan ZA, Manukyan GP, et al. 2008. Predominant role of host genetics in controlling the composition of gut microbiota. PLoS One 3:e3064.

Koenig JE, Spor A, Scalfone N, et al. 2011. Succession of microbial consortia in the developing infant gut microbiome. Proc Natl Acad Sci USA 108:4578-4585.

Konya T, Koster B, Maughan H, et al. 2014. Associations between bacterial communities of house dust and infant gut. Environ Res 131:25-30.

Kuhle S, Tong OS, Woolcott CG. 2015. Association between caesarean section and childhood obesity: a systematic review and meta-analysis. Obesity Rev 16:295-303.

Laursen MF, Zachariassen G, Bahl MI, et al. 2015. Having older siblings is associated with gut microbiota development during early childhood. BMC Microbiol 15:154.

Lax S, Smith DP, Hampton-Marcell J, et al. 2014. Longitudinal analysis of microbial interaction between humans and the indoor environment. Science 345:1048-1052.

Lewis ZT, Totten SM, Smilowitz JT, et al. 2015. Maternal fucosyltransferase 2 status affects the gut bifidobacterial communities of breastfed infants. Microbiome 3:13.

Ly NP, Litonjua A, Gold DR, Celedon JC. 2011. Gut microbiota, probiotics, and vitamin D: interrelated exposures influencing allergy, asthma, and obesity? J Allergy Clin Immunol 127:10871094.

MacIntyre DA, Chandiramani M, Lee YS, et al. 2015. The vaginal microbiome during pregnancy and the postpartum period in a European population. Sci Rep 5:8988. 
Macpherson AJ, Harris NL. 2004. Interactions between commensal intestinal bacteria and the immune system. Nat Rev Immunol $4: 478-485$.

Marietta E, Rishi A, Taneja V. 2015. Immunogenetic control of the intestinal microbiota. Immunology 145:313-322.

Mayer EA, Tillisch K, Gupta A. 2015. Gut/brain axis and the microbiota. J Clin Invest 125:926-938.

Murphy K, CA, Ryan OS, Dempsey CA, et al. 2015. The gut microbiota composition in dichorionic triplet sets suggests a role for host genetic factors. PLoS One 10:e0122561.

Nobel YR, Cox LM, Kirigin FF, et al. 2015. Metabolic and metagenomic outcomes from early-life pulsed antibiotic treatment. Nat Commun 6:7486.

Okogbule-Wonodi AC, Gross GW, et al. 2011. Necrotizing enterocolitis is associated with urea plasma colonization in preterm infants. Pediatr Res 69:442-447.

Pacheco AR, Barile D, Underwood MA, Mills DA. 2015. The impact of the milk glycobiome on the neonate gut microbiota. Annu Rev Anim Biosci 3:419-445.

Palmer C, Bik EM, DiGiulio DB, et al. 2007. Development of the human infant intestinal microbiota. PLoS Biol 5:e177.

Pantoja-Feliciano IG, Clemente JC, et al. 2013. Biphasic assembly of the murine intestinal microbiota during early development. ISME J 7:1112-1115.

Penders J, Gerhold K, Stobberingh EE, et al. 2013. Establishment of the intestinal microbiota and its role for atopic dermatitis in early childhood. J Allergy Clin Immunol 132:601-607.

Penders J, Gerhold K, Thijs C, et al. 2014. New insights into the hygiene hypothesis in allergic diseases: mediation of sibling and birth mode effects by the gut microbiota. Gut Microbes 5:239-244.

Penders J, Thijs C, van den Brandt PA, et al. 2007. Gut microbiota composition and development of atopic manifestations in infancy: the KOALA Birth Cohort Study. Gut 56:661-667.

Penders J, Thijs C, Vink C, et al. 2006. Factors influencing the composition of the intestinal microbiota in early infancy. Pediatrics 118:511-521.

Perez PF, Dore J, Leclerc M, et al. 2007. Bacterial imprinting of the neonatal immune system: lessons from maternal cells? Pediatrics 119:e724-e732.

Petnicki-Ocwieja T, Hrncir T, Liu YJ, et al. 2009. Nod2 is required for the regulation of commensal microbiota in the intestine. Proc Natl Acad Sci USA 106:15813-15818.

Petra L, Georgina LH, Harry JF. 2014. The gut microbiota, bacterial metabolites and colorectal cancer. Nat Rev Microbiol 12:661-672.

Rajilic-Stojanovic M, Jonkers DM, Salonen A, et al. 2015. Intestinal microbiota and diet in IBS: causes, consequences, or epiphenomena? Am J Gastroenterol 110:278-287.
Rausch P, Rehman A, Kunzel S, et al. 2011. Colonic mucosaassociated microbiota is influenced by an interaction of Crohn disease and FUT2 (Secretor) genotype. Proc Natl Acad Sci USA 108:19030-19035.

Robson M, Hartigan L, Murphy M. 2013. Methods of achieving and maintaining an appropriate caesarean section rate. Best Pract Res Clin Obstetrics Gynaecol 27:297-308.

Rogier EW, Frantz AL, Bruno ME, et al. 2014. Secretory antibodies in breast milk promote long-term intestinal homeostasis by regulating the gut microbiota and host gene expression. Proc Natl Acad Sci USA 111:3074-3079.

Romero R, Hassan SS, Gajer P, et al. 2014. The composition and stability of the vaginal microbiota of normal pregnant women is different from that of non-pregnant women. Microbiome 2:4.

Salminen S, Gibson GR, McCartney AL, Isolauri E. 2004. Influence of mode of delivery on gut microbiota composition in seven year old children. Gut 53:1388-1389.

Salzman NH, Hung K, Haribhai D, et al. 2010. Enteric defensins are essential regulators of intestinal microbial ecology. Nat Immunol 11:76-83.

Savino F, Roana J, Mandras N, et al. 2011. Faecal microbiota in breast-fed infants after antibiotic therapy. Acta Paediatr 100:7578.

Smith SM, Smucny J, Fahey T. 2014. Antibiotics for acute bronchitis. JAMA 312:2678-2679.

Stark PL, Lee A. 1982. The microbial ecology of the large bowel of breast-fed and formula-fed infants during the first year of life. J Med Microbiol 15:189-203.

Stewart JA, Chadwick VS, Murray A. 2005. Investigations into the influence of host genetics on the predominant eubacteria in the faecal microflora of children. J Med Microbiol 54:12391242 .

Stokholm J, Schjorring S, Eskildsen CE, et al. 2014. Antibiotic use during pregnancy alters the commensal vaginal microbiota. Clin Microbiol Infect 20:629-635.

Totten SM, Zivkovic AM, Wu S, et al. 2012. Comprehensive profiles of human milk oligosaccharides yield highly sensitive and specific markers for determining secretor status in lactating mothers. J Proteome Res 11:6124-6133.

Trasande L, Blustein J, Liu M, et al. 2013. Infant antibiotic exposures and early-life body mass. Int J Obesity 37:16-23.

Turnbaugh PJ, Hamady M, Yatsunenko T, et al. 2009. A core gut microbiome in obese and lean twins. Nature 457:480-484.

Turnbaugh PJ, Ley RE, Hamady M, et al. 2007. The human microbiome project. Nature 449:804-810.

Turnbaugh PJ, Ley RE, Mahowald MA, et al. 2006. An obesityassociated gut microbiome with increased capacity for energy harvest. Nature 444:1027-1031. 
van Best N, Jansen PL, Rensen SS. 2015. The gut microbiota of nonalcoholic fatty liver disease: current methods and their interpretation. Hepatol Int 9:406-415.

Van de Merwe JP, Stegeman JH, Hazenberg MP. 1983. The resident faecal flora is determined by genetic characteristics of the host. Implications for Crohn's disease? Antonie Van Leeuwenhoek 49:119-124.

Viertel TM, Ritter K, Horz HPP. 2014. Viruses versus bacteria-novel approaches to phage therapy as a tool against multidrug-resistant pathogens. J Antimicrob Chemother 69: $2326-2336$

Volker M, Quintece MM, Rashmi S, Michael G. 2009. Associations between dietary habits and body mass index with gut microbiota composition and fecal water genotoxicity: an observational study in African American and Caucasian American volunteers. Nutr J 8:49.

West CE, Renz H, Jenmalm MC, et al. 2015. The gut microbiota and inflammatory noncommunicable diseases: associations and potentials for gut microbiota therapies. J Allergy Clin Immunol 135:3.
Yatsunenko T, Rey FE, Manary MJ, et al. 2012. Human gut microbiome viewed across age and geography. Nature 486:222-227.

Ye J, Betran AP, Guerrero Vela M, et al. 2014. Searching for the optimal rate of medically necessary cesarean delivery. Birth 41:237-244.

Zhang Z, Geng J, Tang X, et al. 2014. Spatial heterogeneity and co-occurrence patterns of human mucosal-associated intestinal microbiota. ISME J 8:881-893.

Zivkovic AM, German JB, Lebrilla CB, Mills DA. 2011. Human milk glycobiome and its impact on the infant gastrointestinal microbiota. Proc Natl Acad Sci USA 108:4653-4658.

Zoetendal EG, Akkermans ADL, Akkermans-van Vliet WM, et al. 2001. The host genotype affects the bacterial community in the human gastrointestinal tract. Microb Ecol Health Dis 13 :129-134.

Zoetendal EG, von Wright A, Vilpponen-Salmela T, et al. 2002. Mucosa-associated bacteria in the human gastrointestinal tract are uniformly distributed along the colon and differ from the community recovered from feces. Appl Environ Microbiol 68: 3401-3407. 